\title{
World Congress on Osteoporosis, Osteoarthritis and Musculoskeletal Diseases (WCO-IOF-ESCEO 2016): Educational Lecture Abstract
}

(C) International Osteoporosis Foundation and National Osteoporosis Foundation 2016

\section{EL1}

THE ABC OF WRITING A MANUSCRIPT E. Seeman ${ }^{1}$

${ }^{1}$ Endocrine Centre, Austin Health, University of Melbourne, Melbourne, Australia

Don't rush - Script requires full engagement and so, the tranquility of unhurried thought. There must be no urgency of 'tomorrow'.

Title - Later. You are not yet worthy.

Hypotheses - Write these first. Without them you are a rudderless ship on a sea of chaos. The hypotheses are a sextant, the coordinates on the map of scholarship. State the hypotheses in quantitative declarative terms from the most to the least important. Rework them until they accurately capture your vision. Abstract - Write the rationale, hypothesis, method, results and conclusion of the abstract in less than 250 words. If you can do this, write the manuscript.

Introduction - This is challenging. It is not a literature review. It is the rationale - the reasoning upon which the hypotheses are built. If the rationale is flawed, the hypotheses will be. The reasoning depends on critical appraisal of the methodological rigor of the literature. Scientific progress and your contribution depend on this. Never accept authors' inferences. Be wary of your own. Mention and reference the literature upon which your reasoning is based so readers understand what is not known, and so understand the hypotheses but provide critical analyses of this literature in the Discussion, not here.
Methods - Follow the order of the hypotheses. Do not introduce investigations irrelevant to the hypotheses.

Results - Follow the order of the hypotheses. Do not interpret them.

\section{Discussion paragraphs}

1 - Present findings in the order of the hypotheses. Do not interpret them.

2-4 - Now analyze and interpret findings. Are the hypotheses supported by the data? Reconcile the work with the literature. Are inconsistencies the result of flawed or differences in study design, execution or both. If you speculate, say so.

5 - State the limitations of the work - if you don't, others will.

6 - Summary and conclusion. Capture the lesson, usually one message. The conclusion is not a summary. Infer, make a courageous, but wise leap.

Now, the title - If you understand the work you can write the title in under 15 words.

Rework the manuscript - until you do not need to change a word. Only Shakespeare can write a sonnet in one sitting.

Read out loud slowly - Silent reading is lazy, you will ignore what you think is right.

Covering letter - If you understand the work you can write the cover letter to the editor in under 50 words.

Love is blind - Ask a colleague to read the work critically.

Press submit - Remember your name. It is your fathers' before you and your sons after you. One press and a thousand tears won't erase a word. 